\title{
Relationship between measurement uncertainty and verifiability of geometric specifications: the case study of drilled hole orthogonality
}

\author{
D. Vetturi, M. Lancini`, I. Bodini, and S. Pasinetti \\ Department of Mechanical and Industrial Engineering, University of Brescia, Brescia, Italy
}

Received: 22 October 2012 / Accepted: 22 November 2012

\begin{abstract}
In mechanical design, geometrical specifications and dimensional tolerances are commonly used to avoid final product malfunction and to allow for assembly integration. Geometric specification usage, in particular, has many manufacturing and durability implications, the feasibility of their measurement and verification, however, is often neglected and the influence of measurement uncertainty in their evaluation underestimated. Often geometrical specifications are defined without considering measurement uncertainties, or measurability at all: it is not uncommon to find approved specifications prescribing unverifiable geometry, or dimension tolerances that exceed state-of-art measurements. This article explores the case study of orthogonality between a circular hole and the plane on which it is drilled, evaluated using a Coordinate Measuring Machine. Such specification is defined, according to ISO 14253, as the angle between the plane normal and cylinder axis. Uncertainty of points coordinates obtained can, however small, play a key role in the final evaluation of orthogonality: if the specified tolerance is thigh enough it is also possible to have misalignment uncertainty higher than the tolerance itself. The authors propose the results of a mathematical and numerical model, meant to help the designer to define specification to assess the relationship between cylinder-plane misalignment measurability, CMM uncertainty and features dimensions.
\end{abstract}

Keywords: Uncertainty; geometric specification; orthogonality; tolerances verification; measurability

\section{Introduction}

Given that every measurement comes with an associated uncertainty [1-5], it clear how such uncertainty plays a key role in many industrial activities. Quality control and acceptance could be, in particular, source for quarrel whereas uncertainty in measurements on both sides is not taken into account. EN ISO 14253:2011 standard, concerning "Inspection by measurement of workpieces and measuring equipment" [6] focuses on the problem and on its solution, especially in its part two: "Guidelines for achieving agreements on measurement uncertainty statements" [7].

Please consider the common case of a dimensional tolerance for a particular design feature: quality control after production, as well as acceptance controls of the customer, will focus on the measurement of such a dimension to verify its compliance with the prescription [8]. Supposing a bilateral tolerance, that is both upper specification limit $(U S L)$ and a lower specification limit $(L S L)$ are used to define an acceptance region.

Given uncertainty in the dimension measurement, acceptance could be cleared only if the whole confidence interval (a standard value of $k=2$ for the coverage factor

^ Correspondence: matteo.lancini@ing.unibs.it is assumed [7]) associated with the result is within the acceptance region. On the other side, non-conformity could be proven by the recipient only if acceptance region and confidence interval are not overlapping.

These simple consideration lead to the suggestion, given by the ISO standard, of a new definition for both supplier and consumer taking into account measurement uncertainty, where the supplier shall reduce the quality control range by adding uncertainty to $L S L$ and reducing $U S L$ of the same amount. Vice-versa, the acceptance interval will be expanded by the recipient to reduce the non-conformity range of a value equal to the measurement extended uncertainty.

The result of such a procedure is displayed in Figure 1, where it can be noticed how there are no more two but three distinct regions: to the acceptance and refusal interval a gray region, the ambiguity interval, has been added.

The acceptance and refusal regions define the certainty of the piece being complying or not, respectively, with specifications, the ambiguity interval identifies a range of values associated with an uncertain result: the compliance cannot be granted, nor can the non-conformity be proven, leading to the manufacturer discarding the item and the recipient accepting it. 


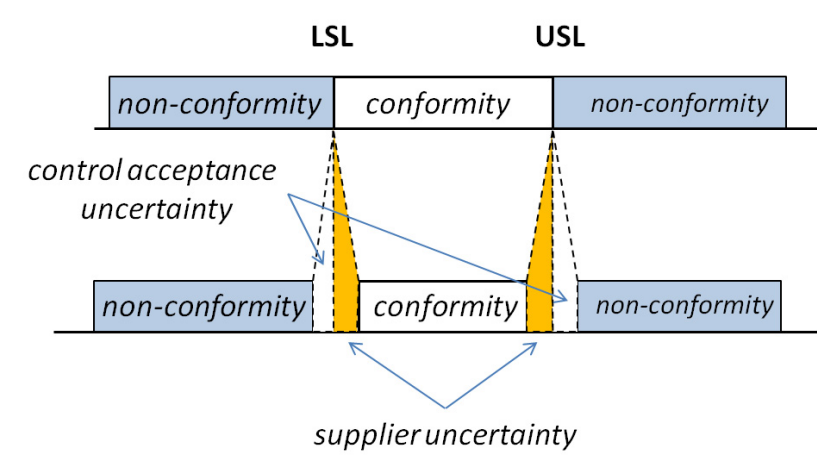

Fig. 1. Acceptance, refusal and ambiguity intervals [7].

These ranges limits are not fixed such in the standard tolerance specifications, but they depend on the measurement accuracy and on its uncertainty assessment.

It is clear that such a procedure involves a higher risk for the manufacturer to discard a suitable product and for the client to accept a unapt piece; however this approach reduces quarrels between the parties. It is also evident that in this way uncertainty assessment plays a major part in the economic sale process, reducing these aforementioned risks.

\section{Model definition for the orthogonality specification verification}

To evaluate the orthogonality between a reference plan and a cylindrical element a mathematical model, in order to assess both misalignement and its uncertainty, has been defined [9].

Such a model takes root in the estimate of geometrical entities (plane and cylinder) using Least Square Method (LSM) and points coordinates measured by a Coordinate Measuring Machine (CMM).

The reference plane $\pi$ is defined using a point $C_{0}$ belonging to the plane itself and its normal versor $\vec{n}_{\text {plane, }}$, so the definition for $\pi$ is a function of some parameters:

$$
\pi=f_{\text {plane }}\left(C_{0}, \vec{n}_{\text {plane }}\right) .
$$

LSM approach evaluates those parameters, as displayed by Figure 2, and minimizing the quantity

$$
\sum_{i}\left[\overrightarrow{P_{i} C_{0}} \cdot \vec{n}_{\text {plane }}\right]^{2}=g_{\text {plane }}\left(C_{0}, \vec{n}_{\text {plane }}\right)=\min
$$

given $P_{i}$ the $i$ th point measured on the plane. The position of those points is uniform in the rectangular domain $L_{x}-L_{y}$.

That minimization procedure can be reduced to a linear least square problem.

Analysis shows that the main cause of the variability of plane direction in order to the point's positions uncertainty is its extension $L_{x}-L_{y}$, as shown in Figure 3.

In a similar way the cylinder is defined, as can be seen in Figure 3, thanks to a point $C_{0}$ belonging to its axis, its

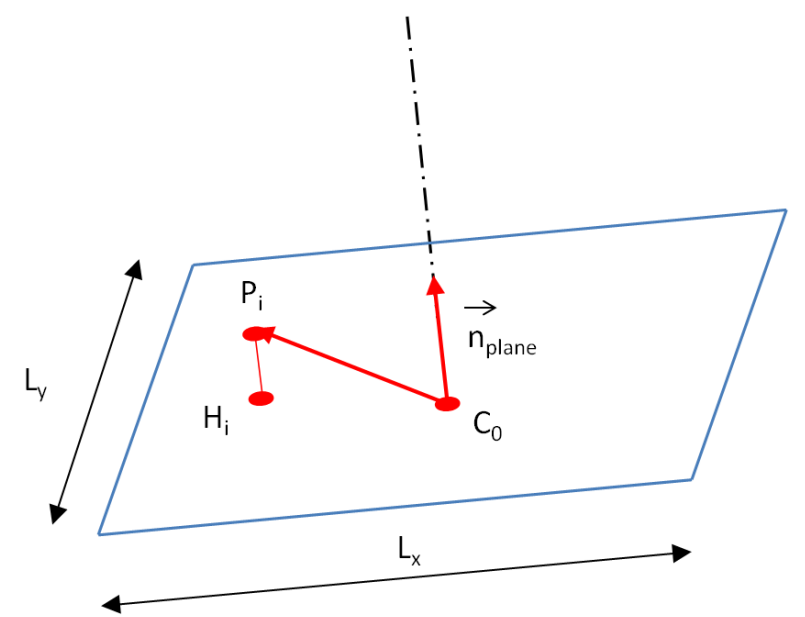

Fig. 2. Plane definition.

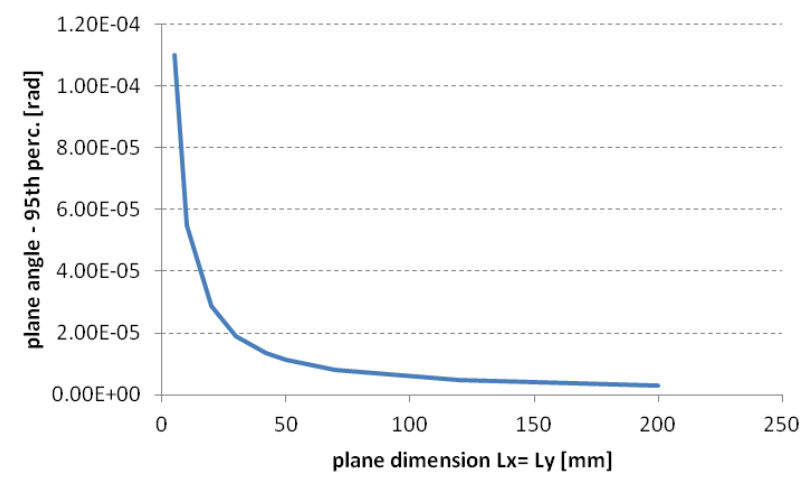

Fig. 3. Plane orientation uncertainty.

axis direction $\vec{v}_{\mathrm{cyl}}$ and its radius value $R$, so the definition for it is a function of that parameters:

$$
\gamma=f_{\mathrm{cil}}\left(C_{0}, \overrightarrow{v_{\mathrm{cil}}}, R\right)
$$

that are obtained by minimizing the quantity

$$
\sum_{i}\left[\overline{P_{i} H_{i}}-R\right]^{2}=g_{\text {cil }}\left(C_{0}, \overrightarrow{v_{\text {cil }}}, R\right)=\min
$$

where $P_{i}$ are points measured on the cylinder, $H \backslash_{i}$ are the projection on cylinder axis of the measured points.

That minimization procedure is a non-linear least square problem which needs a particular procedure for solving.

The main cause of the variability of cylinder direction in order to the point's positions uncertainty is the cylinder height $H$, as shown in Figure 5 .

Deviation from orthogonality, following a common application of Geometrical Product Specification standard, could be expressed as an angle between plane normal versor $\vec{n}_{\text {plane }}$ and cylinder axis versor $\vec{v}_{\text {cyl }}$, time the cylinder height, leading to a definition of misalignment as:

$$
t=H \sin (\alpha)
$$

where $H$ is the cylinder height (see Fig. 6). 


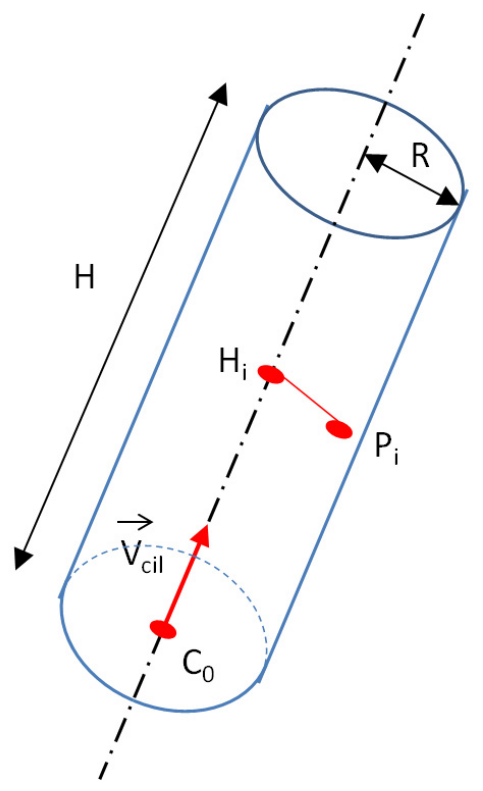

Fig. 4. Cylinder definition.

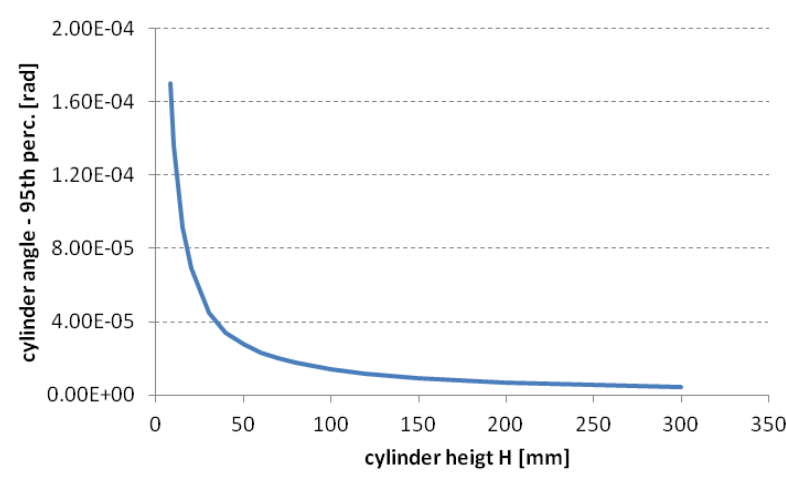

Fig. 5. Cylinder orientation uncertainty.

The variation of misalignment $t$ by cylinder height $H$ and plane dimension $L_{x}-L_{y}$ is a combination of plane and cylinder orientation uncertainty. How it depends on geometrical parameters will be discuss in next paragraphs.

To assess the influence of coordinates measurement uncertainty on orthogonality each point has been associated with a uniform distribution in its coordinates (a spherical spatial distribution coherent with the CMM accuracy) having half-width of $1 \mu \mathrm{m}$ and Monte Carlo simulations were run evaluating $t$ and $\alpha[10,11]$.

\section{Monte Carlo results}

Both the misalignment $t$ and the angle $\alpha$, resulting from simulations, follow a non-negative, asymmetric distribution, an example of the which could be seen in Figure 7 , which describes the uncertainty associated with orthogonally estimate. Of these distributions the 5th, 50th and 95th percentile were taken into account as uncertainty estimators. Since the simulation was based on a perfect alignment case, the 95th percentile describes the
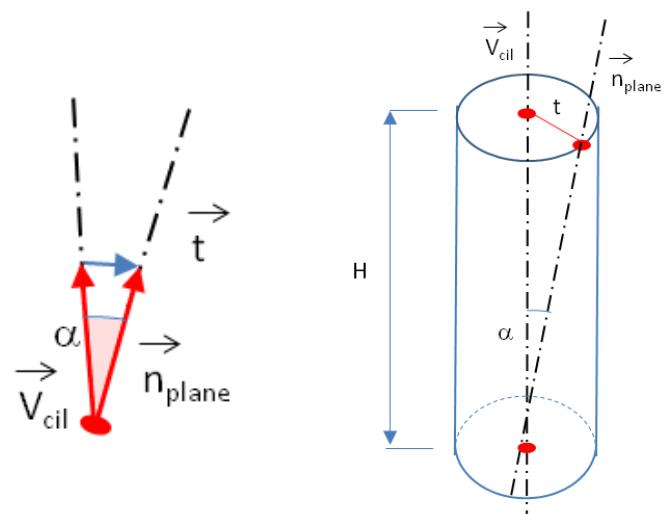

Fig. 6. Misalignment $t$ and versors angle $\alpha$.

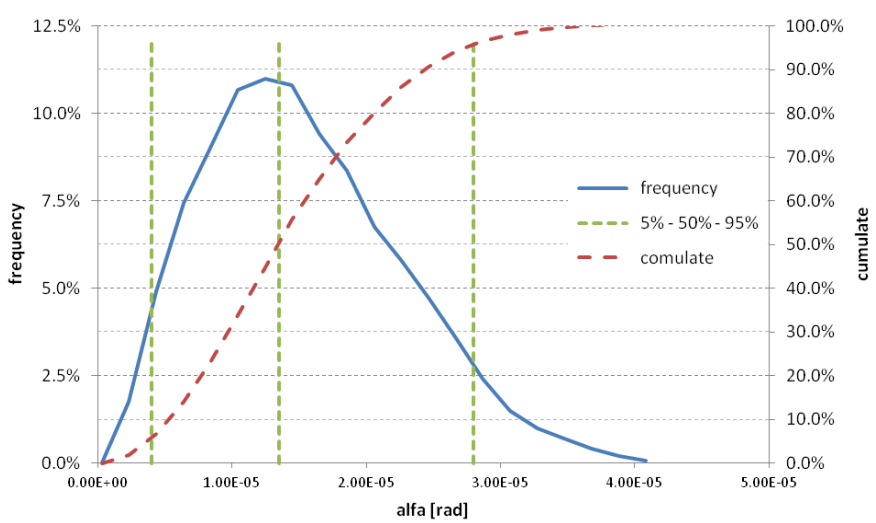

Fig. 7. Orthogonality $t$ and angle $\alpha$, result of $10000 \mathrm{MCM}$ simulations for the case $R=8 \mathrm{~mm}, H=70 \mathrm{~mm}, L_{x}=L_{\mathrm{y}}=$ $30 \mathrm{~mm}$.

extended uncertainty of misalignment with a $95 \%$ confidence level [12].

Different Monte Carlo analysis were undertaken to estimate the influence of geometrical entities (plane and cylinder) on misalignment uncertainty. In particular a rectangular, limited plane, with varying dimensions $L_{x}$ and $L_{y}$, has been taken into account, considering only 9 points on its surface; similarly, the cylinder has been simulated with different radiuses $R$ and heights $H$, considering 8 points measured on its surface by the CMM.

Uncertainty for each point taken by the CMM is considered fixed and unary ( $1 \mu \mathrm{m}$ half-width uniform) in order to facilitate tests results comparisons.

Figure 8 shows how misalignment angle $\alpha$ depends on both plane dimensions (continuous line) and on cylinder height (dotted line): it is clear how with small values of $L_{x}$ plane size play a bigger role in the uncertainty budget, on the other hand, when plane dimension is bigger than cylinder radius, misalignment almost wholly depends on cylinder size.

In the latter case (continuous line), orthogonality uncertainty depends on cylinder height following a linear direct proportionality. 


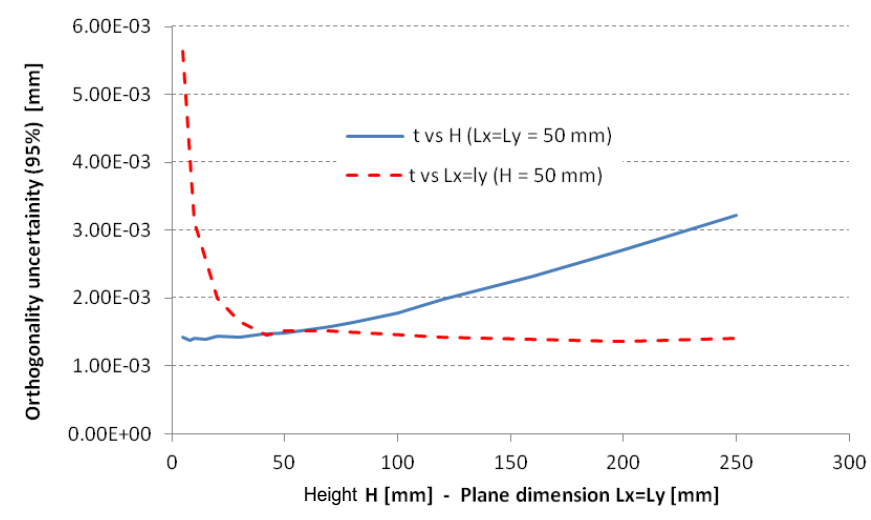

Fig. 8. Versor angle $\alpha$ uncertainty.

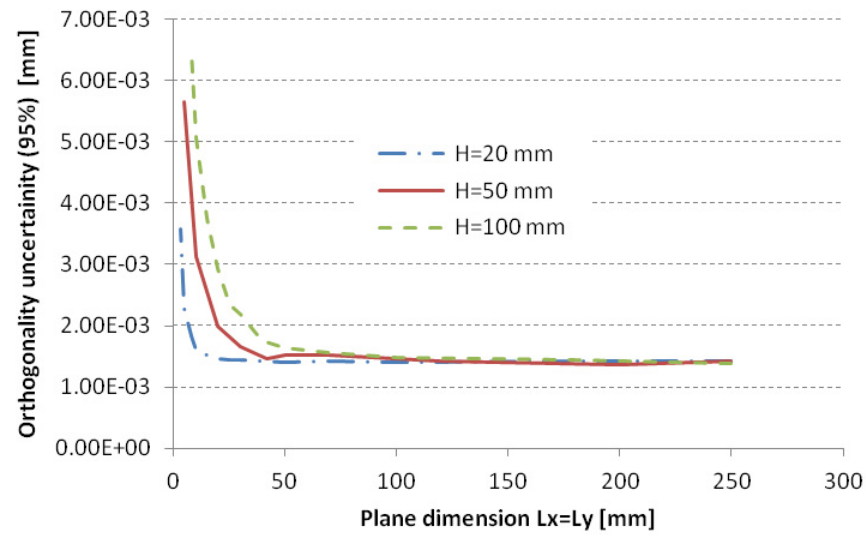

Fig. 9. Angle $\alpha$ uncertainty (95\% percentile plotted).

As can be noticed in Figure 9, plane dimensions contribution to orthogonality uncertainty is higher where the size is smaller, leading to vertical asymptote in zero.

This proves how the plane size allows other contributors (such as cylinder geometry or CMM accuracy) to be relevant only for over a dimension threshold, thus defining a lower limit for misalignment uncertainty which scarcely depends on cylinder height.

Results of these simulations are shown in Figure 10, where two parameters influence on uncertainty was explored: $95 \%$ orthogonality uncertainty is displayed in function of height $H$ and for different dimensions of the plane.

As can be noticed, the cylinder height plays a key role in orthogonality uncertainty: a high value, while improving accuracy in the cylinder orientation measurement, could in fact amplify uncertainty associated with plane orientation.

On the other side, for smaller values of height misalignment uncertainty is wholly dependent on CMM uncertainty associated with point coordinates, up to a point where dimensions of either plane of cylinder are relevant no more.

Plane dimension have also an effect on orthogonality measurement: as can be noticed by Figure 10, only if these dimensions are small enough cylinder height has some effect on uncertainty, otherwise the CMM accuracy is predominant and leads to a lower limit of measurability.

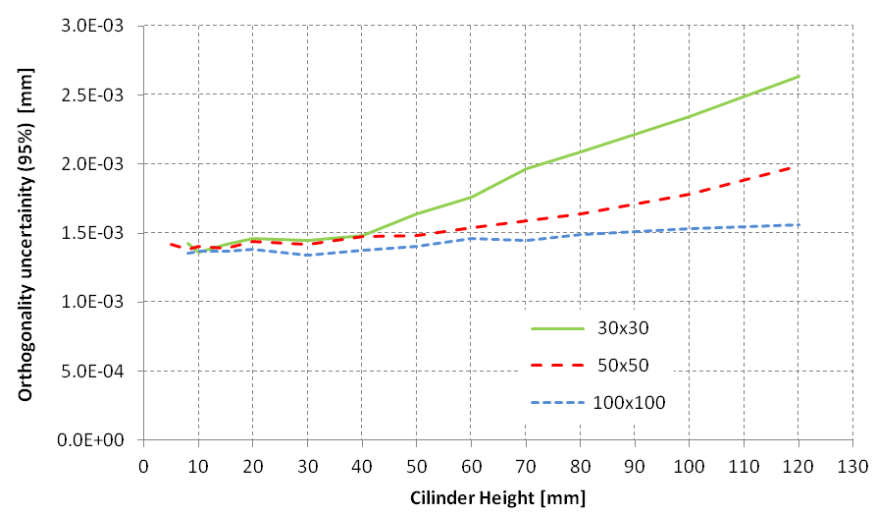

Fig. 10. Orthogonality uncertainty in different geometrical configurations ( $R=8 \mathrm{~mm}, L_{x}=L_{y}$ displayed on chart legend).

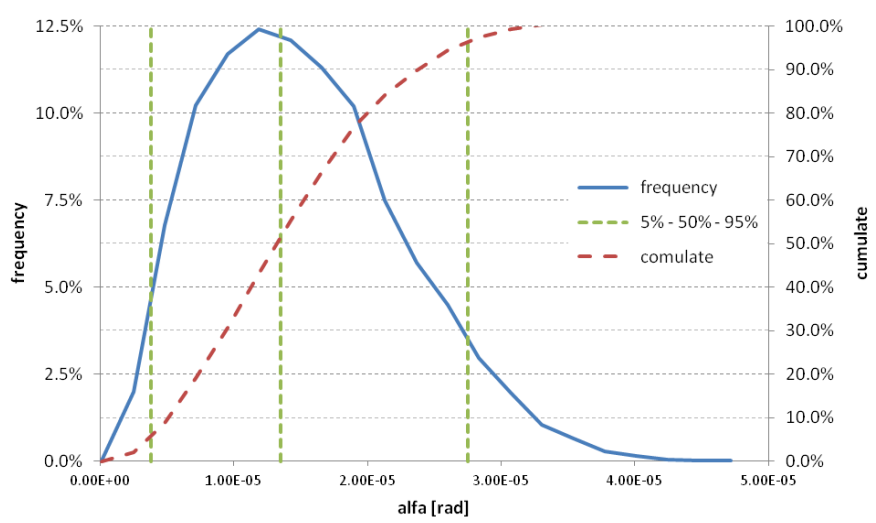

Fig. 11. Angle $\alpha$, result of $10000 \mathrm{MCM}$ simulations for the case $R=20 \mathrm{~mm}, H=70 \mathrm{~mm}, L_{x}=L_{y}=30 \mathrm{~mm}$.

Radius of the cylinder displayed no significant influence on uncertainty, as can be notice on Figure 11, where $\alpha$ obtained using a radius $R=20 \mathrm{~mm}$ has a distribution compatible, in terms of 5 th, 50 th and 95 th percentile, with the one depicted in Figure 9, obtained using $R=8 \mathrm{~mm}$ therefore its influence has been no further explored.

\section{Dimensional normalization}

To describe in more general terms how plane-cylinder orthogonality uncertainty depends on dimensions involved, a geometrical normalization has been proposed, in the reference case of $L_{x}=L_{y}$, defining the ratio between $H$, cylinder height, and $L_{x}$, plane dimension.

$$
\lambda=H / L_{x}
$$

To further synthesize results misalignment uncertainty has been estimated thanks to the 95 th percentile of $t$ population, normalized using as a reference the half-width $\Delta$ of CMM coordinates distribution, obtaining:

$$
\delta=t_{95 \%} / \Delta .
$$

Results of this normalization can be observed in Figure 12 where $\delta$ is plotted as function of $\lambda$, making evident how 


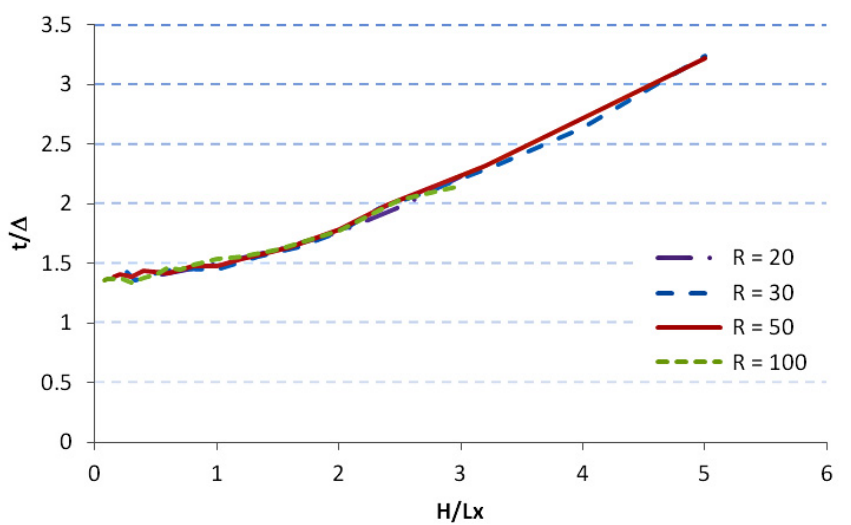

Fig. 12. Normalized uncertainty vs. normalized dimension.

is the ratio between principal dimensions $\left(H\right.$ and $\left.L_{x}\right)$ to define orthogonality uncertainty, thus measurability, while the radius $R$ has a negligible contribution.

\section{Conclusions}

Often quantitative expression of limits describing geometric product specifications is managed by the mechanical designer, which is mainly, sometimes only, concerned about functional aspects of the project. In precision mechanics this leads to very narrow acceptance intervals to guarantee functional performances of the product, however, measurability cannot be always achieved due to the same properties of the item.

This work underlines how geometrical parameters, such as dimensions of the feature involved, can act as magnifiers for measurement uncertainty when verifying a geometric specification: there could be cases where this uncertainty magnification effect could take uncertainty in the measurement at a value equal or higher than the measurement estimate itself, leading to non-measurability of misalignment and non-verifiability of the geometrical specification requested.

It is belief of the authors that mechanical designers should be aware of such a limit, on its dependence on geometrical features, and take it into account when drafting specifications.

\section{References}

1. BIPM, IEC, IFCC, ILAC, IUPAC, IUPAP, ISO, OIML (2012), The international vocabulary of metrology - basic and general concepts and associated terms (VIM), 3rd edn. (JCGM 200:2012)

2. C.F. Dietrich, Uncertainty, Calibration and Probability: the Statistics of Scientific and Industrial Measurement (Adam Hilger, 1991)

3. Evaluation of measurement data - Guide to the expression of uncertainty in measurement JCGM 100:2008 (GUM 1995 with minor corrections)

4. NIST Technical Note 1297, Guidelines for Evaluating and Expressing the Uncertainty of NIST Measurement Results (NIST, 1994)

5. Evaluation of measurement data - An introduction to the "Guide to the expression of uncertainty in measurement" and related documents (JCGM 104:2009)

6. EN ISO 14253-2:2011, Geometrical product specifications (GPS) - Inspection by measurement of workpieces and measuring equipment - Part 2: Guidance for the estimation of uncertainty in GPS measurement, in calibration of measuring equipment and in product verification (ISO 2011)

7. EN ISO 14253-3:2011, Geometrical product specifications (GPS) - Inspection by measurement of workpieces and measuring equipment - Part 2: Guidelines for achieving agreements on measurement uncertainty statements (ISO 2011)

8. EN ISO 9000:2005, Quality management systems Fundamentals and vocabulary (ISO 2005)

9. E.O. Doebelin, Measurement Systems: Application and Design (McGraw-Hill, 2004)

10. Evaluation of measurement data - Supplement 1 to the "Guide to the expression of uncertainty in measurement" Propagation of distributions using a Monte Carlo method (JCGM 101:2008)

11. Evaluation of measurement data - Supplement 2 to the "Guide to the expression of uncertainty in measurement" - Extension to any number of output quantities (JCGM 102:2011)

12. W.W. Hines, D.C. Montgomery, Probability and Statistics in Engineering and Management Science (Wiley, 1990) 